\title{
CJEM Debate Series: \#PoCUS-All physicians practicing emergency medicine should be competent in the use of point-of-care ultrasound
}

\author{
Paul Atkinson, MB, MA*; Paul Olszynski, MD; Jordan Chenkin, MD; Daniel J. Kim, MD; Greg Hall, MD
}

\section{INTRODUCTION}

\section{Paul Atkinson (@eccucourse)}

We continue our series of debate-style editorials with the topic of point-of-care ultrasound (PoCUS). The debate series provides CFEM readers with the opportunity to hear differing perspectives on topics pertinent to the practice of emergency medicine. The debaters have been allocated opposing arguments on topics where there is some controversy or perhaps scientific equipoise.

"Ultrasound is the new stethoscope," we hear from those who promote the use of PoCUS at the bedside. The use of PoCUS is increasing in many medical specialties, including emergency medicine. But does it benefit patient care? Does it improve outcomes? Or is PoCUS simply another tool in the increasingly complex path to diagnosis faced by patients and physicians? Should today's medical students consign their stethoscopes to the archive of historical artefacts, replacing them with handheld ultrasound devices? Or is PoCUS just a passing fad, where new technology promises much but fails to deliver any measurable benefit? Have we reached the point where all physicians practicing modern emergency medicine must demonstrate competency in PoCUS? Or should it remain an optional adjunct for those interested in what it adds to their clinical practice?

Drs. Paul Olszynski and Jordan Chenkin make the argument that PoCUS should now be considered a core competency expectation for all physicians practicing emergency medicine; with Drs. Daniel J. Kim and Greg
Hall, responding to warn us that the evidence and infrastructure supporting PoCUS have not yet reached that tipping point.

Readers can follow the debate on Twitter and vote for either perspective, by going to @CFEMonline or by searching \#CFEMdebate.

\section{\#POCUS - IMPROVED ACCURACY, OUTCOMES, AND} EFFICIENCY IN EMERGENCY CARE

\section{For: Paul Olszynski (@OlszynskiP) and Jordan Chenkin (@POCUS_Toronto)}

With steadily improving portability, accessibility and technology, PoCUS stands to become the most important advance in bedside care in a century. Emergency medicine is at the forefront of this evolution, with both the Canadian Association of Emergency Physicians (CAEP) and the American College of Emergency Physicians (ACEP) publishing guidelines that include over 20 indications for PoCUS use in emergency care. Despite this, questions remain as to whether all emergency physicians (EPs) should be expected to be competent in PoCUS. We argue that the associated positive impacts on clinical assessment, patient outcomes, and system efficiencies make PoCUS a "must-have" skill for all EPs.

Historically, the clinical assessment of patients has been limited to an analysis of information acquired by performing a detailed history of illness, then refined by physical examination providing approximations or

From the *Department of Emergency Medicine, Dalhousie University, Saint John, NB; tDepartment of Emergency Medicine, University of Saskatchewan, Saskatoon, SK; ¥Department of Emergency Medicine, University of Toronto, Toronto, ON; §Department of Emergency Medicine, University of British Columbia, Vancouver, BC; and the IDepartment of Emergency Medicine, McMaster University, Brantford General Hospital, Brantford, ON.

Correspondence to: Paul Atkinson, Department of Emergency Medicine, Dalhousie University, Saint John Regional Hospital, Saint John, NB E2L 4L4, Canada; Email: Paul.Atkinson@Dal.Ca 
indirect measurements of underlying pathophysiologic processes. The physical examination (including earlier uses of sound waves such as auscultation and percussion) was performed with the hope that findings might help confirm or refute the initial suspected diagnoses. Much has changed since the traditional clinical examination was first developed (think evidence-guided medicine and smartphones). We now know that many physical exam findings represent "guesstimates," leaving clinicians with little usable, new information about their patients, and falling far short of meaningful contribution to the diagnosis (or knowing which patients warrant further investigations, or how to proceed when time is of the essence). For example, physical examination findings can be unreliable in patients with suspected pneumonia, deep venous thrombosis (DVT), abdominal aortic aneurysm (AAA), and complicated skin infection, and often fail to reliably determine the correct etiology. ${ }^{1}$ In contrast, meta-analyses examining the reliability of PoCUS findings for these same conditions report substantially more robust results, with sensitivities and specificities for pneumonia at $88 \%$ and $86 \%^{2}$; DVT at $95 \%$ and $96 \%^{3}$; AAA at $99 \%$ and $98 \%{ }^{4}$; and complicated skin infection at $97 \%$ and $83 \%$, respectively. ${ }^{5}$ When these are integrated into the clinician's overall clinical impression the PoCUS findings meaningfully impact the probability of several conditions, thus greatly increasing diagnostic accuracy and the value of the bedside clinical assessment.

In addition to improving diagnostic accuracy, PoCUS can have a direct impact on emergency department (ED) patient outcomes. By arriving at a diagnosis at the bedside, definitive treatment decisions can be made promptly. The use of PoCUS can also reduce unnecessary testing and allow a safer patient discharge from the ED. A prime example of how PoCUS can impact outcomes can be found in patients with ruptured AAA. Most affected patients are not aware that they have an AAA until it ruptures. When this occurs, time is of the essence because rapid diagnosis and early surgical management reduce mortality. ${ }^{6}$ The use of PoCUS in patients with suspected AAA rupture allows the diagnosis to be made in minutes and facilitates an immediate surgical consultation. One study found that the diagnosis of ruptured AAA could be made in less than 6 minutes of arrival in the ED using PoCUS, compared with 83 minutes without PoCUS. ${ }^{7}$ In that study, the use of PoCUS was associated with a total time to the operating room of
12 minutes compared with 90 minutes without PoCUS, and a reduced mortality rate of $40 \%$ compared with $72 \%$.

The use of PoCUS can also have a significant impact on the outcomes of trauma patients by expediting definitive surgical management. In a retrospective review of 49 patients with penetrating cardiac injury, patients who underwent a PoCUS exam had a shorter time to diagnosis and disposition to the operating room (15.5 v. 42.4 minutes), improved neurologic outcomes, and a higher survival rate $(100 \%$ v. $57.1 \%){ }^{8}$ In a randomized controlled trial of 262 blunt and penetrating trauma patients, the use of PoCUS reduced the time to operative care by $64 \%$ from 166 to 57 minutes. ${ }^{9}$

PoCUS can also have a significant impact on the care of patients with a possible ectopic pregnancy. EPs who are able to definitively identify an intrauterine pregnancy can make safer discharge decisions. In a retrospective review, the use of a PoCUS protocol was associated with a reduced rate of discharging patients with an ectopic pregnancy, and a reduced rate of subsequent rupture. ${ }^{10}$ In a retrospective study of patients with a ruptured ectopic pregnancy, the use of PoCUS was associated with a faster time to diagnosis (58 v. 197 minutes), and faster time to operative management (11 v. 322 minutes). ${ }^{11}$

With improved diagnostic accuracy and evidence of improved patient outcomes, it comes as no surprise that there is also a growing body of evidence demonstrating a net positive impact on healthcare efficiency and costs. Several emergency PoCUS applications have been associated with decreased costs of care. Recent studies on the econometric impact of PoCUS on medical and critical care wards ${ }^{12,13}$ show similarly impressive cost savings in the delivery of care. Of note, these economic benefits are borne out relatively quickly, typically within 1 year after the purchase of equipment and integration of PoCUS into routine care.

PoCUS is now widely considered essential to the practice of emergency medicine. It has been almost 20 years since CAEP first issued a position statement supporting the availability of focused ultrasound 24 hours per day in the ED, and nearly 10 years since both the Royal College and College of Family Physicians in Canada included ultrasound as a core competency in their emergency medicine training standards. Countless studies have demonstrated PoCUS to be a versatile, learnable, and safe skill that has a meaningful impact on all levels of patient care - from 
improved diagnostic accuracy to better patient outcomes and increased system efficiency. The time has come for us to expect all EPs to have basic competence in PoCUS and to incorporate it into their practice.

\section{\#POCUS - INADEQUATE ACCESS, TRAINING, AND}

EVIDENCE TO SUPPORT MANDATORY COMPETENCE

\section{Against: Daniel J. Kim (@dan__kim) and Greg Hall (@fullcode93)}

It is both dangerous and premature to insist that all physicians practicing emergency medicine in Canada must demonstrate competency in the use of PoCUS. Such a demand, along with a lack of access to equipment, supplies, training, and high-level evidence to support beneficial patient-centred outcomes, risks causing harm. Being required to perform PoCUS fails to recognize the varying practice patterns, training, and resources available to the broad assortment of EDs that currently serve the Canadian population.

A great lesson can be learned from the history of laparoscopic and minimally invasive surgery. "Video endoscopy was considered an overnight surgical sensation that was 75 years in the making." ${ }^{14}$ There was a very steep learning curve as well as higher complication rates versus the traditional open approaches of that era. The development of improved video technology and techniques was required before laparoscopic surgery could be proven to be of benefit to the patient. If all surgeons were expected to be competent in laparoscopic surgery in the early stages of its development, significant harm would have been introduced to the population.

Likewise, insisting that all EPs require competency in PoCUS when the technology is still rapidly evolving, the accessibility to the appropriate equipment still lacking, and the quality assurance mechanisms absent in all but a few academic centers, is courting disaster by replacing current practice with untested or undertrained PoCUS-based practice.

To designate PoCUS as a core emergency medicine competency, there should be high quality evidence to support this proposal. However, the majority of current PoCUS research is methodologically weak, does not demonstrate clear benefit in patient-oriented outcomes, and, in some cases, actually shows potential harm.

Focused assessment with sonography in trauma (FAST) is considered a key application in both
Canadian and American emergency medicine PoCUS guidelines. ${ }^{15,16}$ However, a Cochrane review of the use of FAST in blunt abdominal trauma revealed that there was no mortality benefit. ${ }^{17}$ A recent pediatric study investigated the use of FAST in hemodynamically stable children who sustained blunt torso trauma. FAST did not improve clinical care, and there was no effect on ED length of stay or rates of missed intra-abdominal injuries. ${ }^{18}$

Studies of lung ultrasound similarly do not demonstrate any clear patient benefit. A clinical trial of patients presenting with respiratory symptoms randomized patients to a usual care group and a PoCUS protocol group involving an ultrasound of the heart, lungs, and deep veins. Although more patients in the PoCUS group had a correct presumptive diagnosis 4 hours into their ED stay, there was no difference in hospital length of stay or mortality. Even more concerning, the ultrasound group discovered additional pathology that led to increased downstream testing and interventions, yet showed no improvement in mortality. ${ }^{19}$

Another lung ultrasound study found that using PoCUS reduced the use of chest X-rays in pediatric patients with suspected pneumonia. However, it increased the number of patients diagnosed with pneumonia and treated with antibiotics with no difference in clinical outcomes. ${ }^{20}$ In other words, PoCUS can lead to an over-diagnosis and interventions that do not provide benefit and may expose patients to harm.

While PoCUS has been promoted as being of particular utility in cardiac arrest, its use has led to unintended consequences. A recent study of PoCUS in cardiac arrest revealed that the use of PoCUS was associated with significantly longer pulse checks. The mean duration of pulse checks in the PoCUS group was 21 seconds compared with 13 seconds in the nonPoCUS group. ${ }^{21}$ This is concerning because current resuscitation guidelines emphasize the importance of the delivery of high quality chest compressions with minimal interruption.

In addition to the lack of compelling evidence, there is the practical issue of limited access to adequate equipment, training, support, and maintenance of skills. PoCUS is an operator-dependent skill requiring significant practice to acquire and maintain proficiency. ${ }^{22}$ Practice requires access to appropriate equipment and supportive infrastructure, which is lacking in many hospitals. These problems are amplified in rural EDs. 
For example, 39\% of rural Ontario physicians report not having an ultrasound machine available in the ED, and $56 \%$ report not knowing how to perform PoCUS. In rural Newfoundland and Labrador, only $4 \%$ of small EDs report having an ultrasound machine. ${ }^{22}$

Our informal survey of approximately 1000 Canadian physicians who had participated in a PoCUS training course listed access to ultrasound equipment as one of the most important barriers to adopting it into practice. To insist that all EPs should be competent in skills, without provision of the tools required to develop or maintain such skills, is nonsensical. It would be comparable to expecting a teenager to become a proficient driver without giving him or her access to a car.

Access to appropriate training for currently practicing physicians is another reason for concern. To achieve competency in many of the PoCUS applications considered fundamental to emergency medicine requires a great deal of supervised practice and instruction. ${ }^{23}$ Beyond introductory courses, there are very few programs outside of fellowships that provide this supervision. Cost and geographic barriers preclude many community and rural EPs from being able to access these programs. For remote practices, the combined costs of a course and travel can be prohibitive, making the achievement of PoCUS competence even more challenging and unfeasible.

Around half of the physicians who staff Canadian EDs are family physicians who do not have specific emergency medicine certification, and this is particularly true of the majority of physicians who provide emergency care in remote and rural settings. ${ }^{24}$ In addition, there is a lack of PoCUS training in residency for family physicians who staff the majority of EDs across Canada. Very few family medicine residencies offer exposure to PoCUS, and almost none provide the amount of practice and training required to practice independently. There is no mention of PoCUS skills in the core family medicine curriculum and training objectives. ${ }^{25}$ So how can we even begin to suggest that all EPs should be competent in PoCUS, if we continue to train most of them through programs that completely ignore this requirement?

If PoCUS is to become an important core competency, we would expect all emergency medicine residency programs to deliver PoCUS training that is standardized in scope. However, the reality is that amongst Canadian emergency medicine training programs, there is "considerable heterogeneity in the scope of ultrasound training, curricula, and determination of proficiency." ${ }^{26}$ It is only within the past year that CAEP's Emergency Ultrasound Committee released a report listing recommended applications for a core PoCUS curriculum. ${ }^{24}$

As useful and practice-changing as PoCUS can be for physicians and their patients, we are not yet ready to declare that all physicians must be competent in performing ultrasound in their emergency medicine practice environments. It makes more sense to declare that the management of particular conditions or performance of certain procedures in a specific environment should be supported by the use of PoCUS. Those who wish to engage in such clinical activities would then be obliged

to acquire and demonstrate competency in ultrasound.

Until we have the evidence to support improved clinical outcomes, the training and equipment to develop proficiency in its application, and the infrastructure to ensure maintenance of competency and quality assurance, PoCUS remains a useful tool for some but not all physicians in emergency medicine.

Keywords: competency, point-of-care ultrasound, standards

Competing interests: PO chairs the CAEP Emergency Ultrasound Committee, GH is Vice President of the Canadian Point-of-Care Ultrasound Society, DJK is the education lead for the CAEP Emergency Ultrasound Committee, and PA is Co-Director of ECCUcourse.com. All authors are involved in medical education that includes PoCUS training.

\section{REFERENCES}

1. Simel DL, Rennie D (eds.) The rational clinical examination: evidence-based clinical diagnosis. New York: McGraw-Hill; 2009.

2. Long L, Zhao HT, Zhang ZY, et al. Lung ultrasound for the diagnosis of pneumonia in adults: a meta-analysis. Medicine (Baltimore) 2017;96:e5713.

3. Rubano E, Mehta N, Caputo W, et al. Systematic review: emergency department bedside ultrasonography for diagnosing suspected abdominal aortic aneurysm. Acad Emerg Med 2013;20:128-38.

4. Burnside PR, Brown MD, Kline JA. Systematic review of emergency physician-performed ultrasonography for lower-extremity deep vein thrombosis. Acad Emerg Med 2008; $15: 493-8$.

5. Subramaniam S, Bober J, Chao J, et al. Point-of-care ultrasound for diagnosis of abscess in skin and soft tissue infections. Acad Emerg Med 2016;23:1298-306. 
6. Hans SS, Huang RR. Results of 101 ruptured abdominal aortic aneurysm repairs from a single surgical practice. Arch Surg 2003;138:898-901.

7. Reed MJ, Cheung LT. Emergency department led emergency ultrasound may improve the time to diagnosis in patients presenting with a ruptured abdominal aortic aneurysm. Eur 7 Emerg Med 2014;21:272-5.

8. Plummer D, Brunette D, Asinger R, et al. Emergency department echocardiography improves outcome in penetrating cardiac injury. Ann Emerg Med 1992;21: 709-12.

9. Melniker LA, Leibner E, McKenney MG, et al. Randomized controlled clinical trial of point-of-care, limited ultrasonography for trauma in the emergency department: the first sonography outcomes assessment program trial. Ann Emerg Med 2006;48:227-35.

10. Mateer JR, Valley VT, Aiman EJ, et al. Outcome analysis of a protocol including bedside endovaginal sonography in patients at risk for ectopic pregnancy. Ann Emerg Med 1996;27:283-9.

11. Rodgerson JD, Heegaard WG, Plummer D, et al. Emergency department right upper quadrant ultrasound is associated with a reduced time to diagnosis and treatment of ruptured ectopic pregnancies. Acad Emerg Med 2001;8:331-6.

12. Testa A, Francesconi A, Giannuzzi R, et al. Economic analysis of bedside ultrasonography (US) implementation in an Internal Medicine department. Intern Emerg Med 2015;10:1015-24.

13. Mitchell D, Leng A, Gardiner S, et al. Using point-of-care bedside ultrasound for volume assessment in early shock: an outcome study. Chest 2016;149:A148.

14. Wetter PA (ed.). Let there be light: a historical analysis of endoscopy's ascension since antiquity. Blog post. Available at: http://laparoscopy.blogs.com/endoscopyhistory/ (accessed 3 October 2017).

15. Olszynski P, Kim D, Chenkin J, et al. The core emergency ultrasound curriculum project: a report from the Curriculum Working Group of the CAEP Emergency Ultrasound Committee. CFEM 2018;20(2):176-82, doi:10.1017/cem. 2017.44 .

16. American College of Emergency Physicians. Emergency ultrasound guidelines. Ann Emerg Med 2009;53:550-70.
17. Stengel D, Rademacher G, Ekkernkamp A, et al. Emergency ultrasound-based algorithms for diagnosing blunt abdominal trauma. Cochrane Database Syst Rev 2015;9: CD004446.

18. Holmes JF, Kelley KM, Wootton-Gorges SL, et al. Effect of abdominal ultrasound on clinical care, outcomes, and resource use among children with blunt torso trauma: a randomized clinical trial. $7 A M A$ 2017;317:2290-6.

19. Laursen CB, Sloth E, Lassen AT, et al. Point-of-care ultrasonography in patients admitted with respiratory symptoms: a single-blind, randomised controlled trial. Lancet Respir Med 2014;2:638-46.

20. Jones BP, Tay ET, Elikashvili I, et al. Feasibility and safety of substituting lung ultrasound for chest x-ray when diagnosing pneumonia in children: a randomized controlled trial. Chest 2016;150:131-8.

21. Huis In 't Veld MA, Allison MG, Bostick DS, et al. Ultrasound use during cardiopulmonary resuscitation is associated with delays in chest compressions. Resuscitation 2017;119:95-8.

22. Micks T, Sue K, Rogers P. Barriers to point-of-care ultrasound use in rural emergency departments. CFEM 2016;18:475-9.

23. Blehar D, Barton B, Gaspari R. Learning curves in emergency ultrasound education. Acad Emerg Med 2015;22: 574-82.

24. Collaborative Working Group on the Future of Emergency Medicine in Canada. Emergency medicine training and practice in Canada: celebrating the past and evolving for the future. Ottawa: The Canadian Association of Emergency Physicians, the College of Family Physicians of Canada, and the Royal College of Physicians and Surgeons of Canada; 2016.

25. Allen T, Bethune C, Brailovsky C, et al. Defining competence for the purposes of certification by the College of Family Physicians of Canada: the evaluation objectives in family medicine. Mississauga: The College of Family Physicians of Canada; 2010.

26. Kim DJ, Theoret J, Liao MM, et al. The current state of ultrasound training in Canadian emergency medicine programs: perspectives from program directors. Acad Emerg Med 2012;19:1073-8. 